\title{
Verification of Crystallite Theory of Glass Modeling Using Rietveld Method
}

\author{
M. R. Ebeid, S. K. Abdelraheem, E. M. Abdel-Minem, K. Abdel-Hady \\ and A. A. Ramadan* \\ Physics Department, Minia Univ., Minia, Egypt \\ *Physics Department, Helwan Univ., Helwan, Cairo, Egypt
}

\begin{abstract}
To verify the validity of the crystallite theory of amorphous models, the phase of $\alpha$-quartz has been chosen for representing the silica glass. The fullpattern fitting of Rietveld method is used as a possible approach to produce artificial amorphous-like powder diffraction patterns, also, the corresponding distance correlation functions were calculated. From comparative study of the characteristics of the obtained XRD patterns and real space correlation functions, it is concluded that the silica glass can be modeled as a strained very small crystals (crystallites) of a polymorph. This can be considered as a useful application of Rietveld method and competent verification of the crystallite theory of glass modeling.
\end{abstract}




\section{Introduction}

Study of the materials in the amorphous state and information about the amorphous structure are of vital importance from the scientific and technological points of view. The crystalline-to-amorphous ratio and the structural information of the amorphous materials, as that of the crystalline one, are essential prerequisites to understand the physical and chemical properties. One of the theories of the amorphous models is the crystallite theory, which envisaged glass as an assembly of very small crystals (crystallites) of the same structure as the corresponding crystalline counterpart. Thus, useful information on the structure of amorphous solids can be obtained from a simple comparison with the corresponding crystalline polymorphs as a starting point for structural models. This does not necessarily mean that the crystallite theory is accepted, but the short-range order may resemble that of the associated crystals [1].

In order to obtain the correct form of diffraction pattern from a crystal based model, it is necessary to include disorder (strain). Le Bail et al.[2] and Le Bail [3] have applied a modified Rietveld program (ARITVE) to refine pure amorphous phases like crystalline ones using microstrained crystalline models. This is based on the fact that, when a crystalline model is distorted mathematically by the application of a statistical isotropic microstrain and/or decreasing the crystallite size (nano-crystallite), an artificial amorphous-like powder diffraction patterns are produced. The method was considered [4] as a variation of the Reverse Monte Carlo (RMC) technique. Also, it can be classified among the methods using periodic boundary conditions [3].

Although, there are many evidence for the existence of such a model, there are still some doubt and questions about its validity. Therefore, this work aims to verify the validity of such crystallite theory of amorphous models by careful inspection of the simulated artificial amorphous-like powder diffraction patterns of silica glass (with different microstrain and crystallite size) and the corresponding total distance correlation function. An Algorithm Based on a reliable line broadening theory [5] will be applied using Warren-Averbach approach [6] to connect the profile with the crystallite size and microstrain.

\section{Method}

\section{Simulated diffraction pattern}

The Rietveld method can be used to simulate artificial powder diffraction patterns [7]. The powder diffraction pattern may be thought of as a collection of individual reflections profiles, each of which has a peak position, a breadth, tails and an integrated area which is proportional to the Bragg 
intensity. Many Bragg reflections contribute to the calculated (simulated) intensity $I_{\text {calc }}(2 \theta)$ at any arbitrary chosen point, $2 \theta$. The first step for the evaluation of $I_{\text {calc }}(2 \theta)$ is the calculation of intensities $I_{\text {calc }}(h k l)$ from the atomic arrangement of the model (unit cell):

$$
\mathrm{I}_{\text {calc }}(\mathrm{hkl})=\mathrm{SL}_{\mathrm{P}}|\mathrm{F}(\mathrm{hkl})|^{2} \text {, }
$$

where $\mathrm{S}$ is a scaling factor and $L_{P}$ contains the Lorentz, polarization and multiplicity factors while the $\mathrm{h}, \mathrm{k}$ and $\mathrm{l}$ are the Miller indices for a Bragg reflection. The structure factor $\mathrm{F}(\mathrm{hkl})$ for any particular (hkl) reflection is calculated from the fractional atomic coordinates of all atoms in the unit cell and their atomic form factors.

The $I_{\text {calc }}(h k l)$ values obtained from Eqn.(1) are subsequently transform into $I_{\text {calc }}(2 \theta)$. This is achieved by adding all those intensities $I_{\text {calc }}(h k l)$ for each of which the $2 \theta(h k l)$ value lies in the interval $(2 \theta-\Delta \theta$, $2 \theta+\Delta \theta$ ), where $\Delta \theta$ depends on the width of a reflection (hkl). Thus,

$$
\mathrm{I}_{\text {calc }}(2 \theta)=\sum_{\mathrm{hkl}} \mathrm{I}_{\text {calc }}(\mathrm{hkl}) \cdot \phi(2 \theta-2 \theta(\mathrm{hkl}))
$$

where $\phi$ is the profile shape function.

In the existing and known programs of Rietveld Method (DBWS [8] or GSAS [9]) and the ARITVE program modified and used by Le Bail [3], the effects of microstructural disorder (crystallite size and microstrain) on the profile broadening were described mathematically by profile shape parameters, for example of the empirical formula of Cagoliote et al. [10] following the original procedure indicated by Rietveld [11]. However, a different view point is adopted in the used program (LS1 [12]). Instead of using phenomenological relations, such as the Caglioti formula, to describe the change of profile broadening as a function of diffraction angle $(2 \theta)$ relationships were derived for profile parameters which are based on a reliable line broadening theory. A model based on the Warren-Averbach approach [6], using conditions on cosine Fourier coefficients and their derivatives, has been adopted to connect the actual profile width and shape directly with the crystallite size and microstrain [5\&13]. The profiles are described [5] by convolution of the true line broadening, $\mathrm{h}_{\mathrm{f}}$, the symmetric, $\mathrm{g}_{\mathrm{s}}$, and asymmetric, $\mathrm{g}_{\mathrm{a}}$, parts of the instrumental function plus the background, $\mathrm{Y}_{\mathrm{b}}$, thus,

$$
\mathrm{h}_{\mathrm{c}}(2 \theta)=\left[\mathrm{h}_{\mathrm{f}}(2 \theta) *\left(\mathrm{~g}_{\mathrm{s}}(2 \theta) * \mathrm{~g}_{\mathrm{a}}(2 \theta)\right)\right]+\mathrm{Y}_{\mathrm{b}}(2 \theta)
$$


The profiles $h_{f}$ and $g_{s}$ have been modeled by pseudo-Voigt function $(p V)$ and $g_{a}$ by an exponential function while the background is reproduced by a polynomial. The shape parameters for the true line broadening are determined by the crystallite size $M$ and root-mean-square (r.m.s) microstrain $\left(E^{2}\right)^{1 / 2}$. They $\left(\mathrm{M}\right.$ and $\left(\mathrm{E}^{2}\right)^{1 / 2}$ ) have been introduced by following the single peak method for Fourier analysis developed by Nandi et al. [14]. The Fourier transform of $\mathrm{pV}$ function $\left(A_{L}\right)$ was subjected to the following condition:

$$
\left(\frac{d A_{L}}{d L}\right)_{L=0}=-\frac{1}{M_{h k l}}
$$

where the column length (normal to the reflecting hkl plane) $L=n d \sqrt{h^{2}+k^{2}+l^{2}}$ and $\mathrm{n}$ and $\mathrm{d}$ are a harmonic number and the interplaner spacing, respectively. The Fourier coefficient $\mathrm{A}_{\mathrm{L}}$ is the product of coefficients for both effects; distortion $A_{L}^{D}$ and crystallite size $A_{L}^{S}$, thus

$$
A_{L}=A_{L}^{D} \cdot A_{L}^{S}
$$

The strain and size Fourier coefficients are connected to $M$ and $\left\langle\varepsilon^{2}\right\rangle^{1 / 2}$, respectively, by

$$
A_{L}^{D}=\exp \left(-\frac{2 \pi^{2}\left\langle\varepsilon_{L}^{2}\right\rangle L^{2}}{2 d^{2}}\right)
$$

and

$$
A_{L}^{S}=K \exp \left(-\frac{L}{M_{h k l}}\right)+\frac{1-K}{2} \exp \left(-\frac{2 L^{2}}{M_{h k l}^{2}}+\frac{1}{2}\right)
$$

It can be seen from Eqn.(6) that the crystallite size distribution, which is proportional to the second derivative of the Fourier Coefficient $A_{L}^{S}$, is a weighted sum of two distributions representing the contribution of Cauchy and that of Gauss component of the profile, where $\mathrm{K}$ is the weight. The above conditions were imposed to the analytical transform of $\mathrm{pV}$ profiles obtaining equations connecting the half width at half maximum (w) and the Gaussian fraction $\eta$ in $\mathrm{pV}$ function to $\mathrm{M},\left(\varepsilon^{2}\right)^{1 / 2}$ and $\mathrm{K}$, which can be solved within the refinement procedure.

Another problem in Rietveld method is the anisotropy of both size and strain where profiles with different indices are broadened in a different manner. An important feature of the present approach is the possibility to introduce the 
anisotropy effect by the description of $M_{h k l}$ and $\left(\varepsilon^{2}\right)^{1 / 2}$ by tensors to account for anisotropy along different crystallographic directions. In the present work, only the isotropic case was enough to be considered.

\section{Correlation function}

The distance correlation function, $\mathrm{T}(\mathrm{r})$, includes merely one dimensional information of the three dimensional atomic distribution of the amorphous materials in the real space. It is the Fourier transform of the total structure factor $(\mathrm{F}(\mathrm{Q}))[15]$. Thus,

$$
\mathrm{T}(\mathrm{r})=4 \pi \mathrm{r} \rho_{0}+\frac{2}{\pi} \int_{0}^{\mathrm{Q}_{\max }}[\mathrm{F}(\mathrm{Q})-1] \mathrm{M}_{\mathrm{th}}(\mathrm{Q}) \sin (\mathrm{Qr}) \mathrm{QdQ}
$$

where $\rho_{0}$ is the average number density of atoms, $\mathrm{Q}$ is the scattering vector $(\mathrm{Q}=4 \pi \sin \theta / \lambda)$ and $\mathrm{Q}_{\max }$ is its upper limit. The function $M_{t h}(Q)=\exp \left(-B_{t h} Q^{2}\right)$ is introduced in order to reduce the termination effect, where $B_{t h}$ is artificial thermal function. The total structure factor, $F(Q)$, is calculated as,

$$
F(Q)=\left\{I(Q)-\left[\left\langle f^{2}(Q)\right\rangle-\langle f(Q)\rangle^{2}\right]\right\} /\langle f(Q)\rangle^{2}
$$

where

$$
\left\langle f^{2}(Q)\right\rangle=\sum_{i=1}^{n} c_{i} f_{i}^{2}, \quad\langle f(Q)\rangle^{2}=\left(\sum_{i=1}^{n} c_{i} f_{i}\right)^{2},
$$

$c_{i}$ is the atomic fraction of $\mathrm{i}$-atoms in the molecule and $f_{i}$ is the $\mathrm{X}$-ray form factor. The $I(Q)$ is the normalized coherent intensity. The Krogh-MoeNorman method was used to normalize the measurement intensities [15]. The distance correlation function, $\mathrm{T}(\mathrm{r})$, includes merely one dimensional information of the three dimensional atomic distribution of the amorphous materials in the real space. The reliability of the structural characteristics obtained from $\mathrm{T}(\mathrm{r})$ reflects the validity of the corresponding diffraction pattern and the used model.

\section{Model of amorphous silica}

The phase of $\alpha$-quartz has been chosen for representing the silica in this study because the similarities in bonding, density and position of the first diffraction peak in amorphous silica and $\alpha$-quartz (crystalline), have led to the suggestion that there is a close structural relationship over short length scales between these two phases (amorphous and crystalline) [16,17].

In order to produce an artificial amorphous-like powder diffraction patterns, $\alpha$-quartz of hexagonal structure $\left(\mathrm{P}_{1} 2\right)$ was used. The structural 
characteristics of the used model [18] are lattice parameters $a=b=0.4913 \mathrm{~nm}$ and $\mathrm{c}=0.5405 \mathrm{~nm}$ and number of molecules per unit cell $\mathrm{z}=3$. The three silicon atoms are placed at positions of $(3 \mathrm{a}):(-\mathrm{u},-\mathrm{u}, 1 / 3),(\mathrm{u}, 0,0)$ and $(0, \mathrm{u}, 2 / 3)$ with $u=0.465$. The other six oxygen atoms are at the positions $(6 c)$ : (xyz) $(y-x,-$ $\mathrm{x}, \mathrm{z}+1 / 3),(-\mathrm{y}, \mathrm{x}-\mathrm{y}, \mathrm{z}+2 / 3),(\mathrm{x}-\mathrm{y},-\mathrm{y},-\mathrm{z}),(\mathrm{y}, \mathrm{x}, 2 / 3-\mathrm{z})$ and $(-\mathrm{x}, \mathrm{y}-\mathrm{x}, 1 / 3-\mathrm{z})$ with $\mathrm{x}=$ $0.415, y=0.272$ and $z=0.125$. Using the given crystallographic information and increasing microstrain and decreasing crystallite size, the simulated diffraction pattern of the amorphous phase was obtained using the LS1 program [12]. From the simulated intensities, the real space correlation function, $T(r)$, has been calculated obeying the line of Wright[1].

\section{Results and Discussion}

To verify the crystallite theory using the simulated diffraction patterns of amorphous structure, the microstrain was varied from 0.01 to 0.06 and crystallite size from 4 to 1 $\mathrm{nm}$, then, the corresponding correlat-ion function, $\mathrm{T}(\mathrm{r})$, was determined at each value. The diffraction patterns and $\mathrm{T}(\mathrm{r})$ for the crystalline form $(\mathrm{M}=50 \mathrm{~nm}$ and $\left.\left(\varepsilon^{2}\right)^{1 / 2}=0.0001\right)$ are shown in Fig.(1). Sharp peak profile for both the XRD and $\mathrm{T}(\mathrm{r})$ is clearly observed. Two series of data were considered. In the first series, the crystallite size was kept constant at $1 \mathrm{~nm}$ while the values of strain are increased from 0.01 to 0.06 and, in the second one, the strain value is kept constant at 0.02 while the crystallite size is
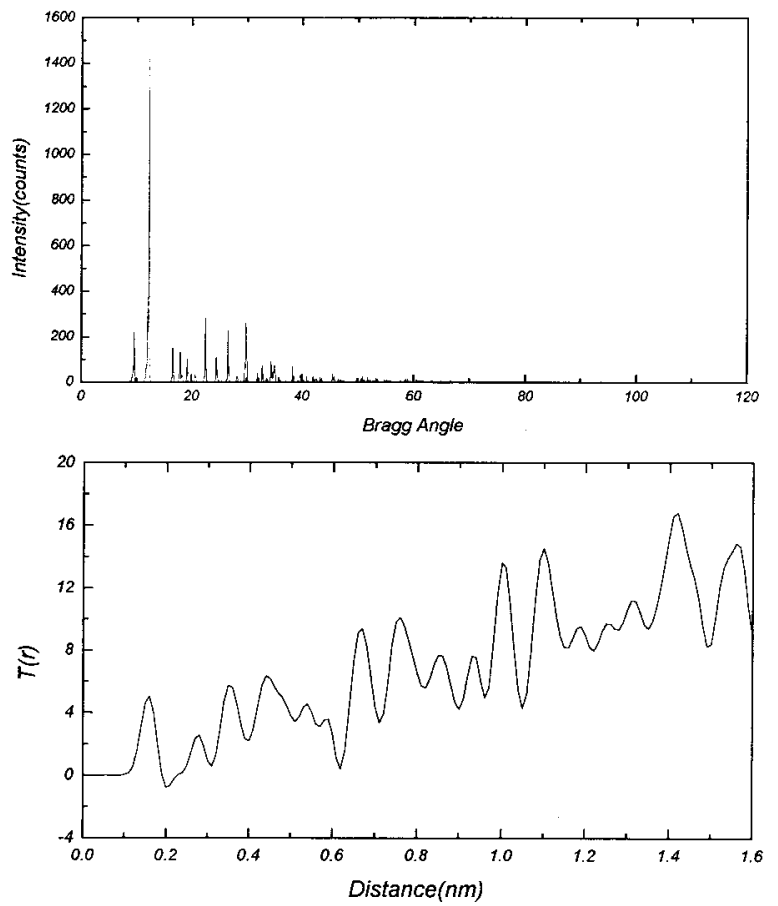

Fig.(1): Simulated XRD pattern and distance correlation function, $T(r)$, of the crystalline form. 
Figures (2\&3) depict the variation of XRD and T(r) at various values of strain and crystallite size, respectively. There are a clear analogy between the $\mathrm{T}(\mathrm{r})$ curves of the artificial amorphous-like and that of their crystalline counter
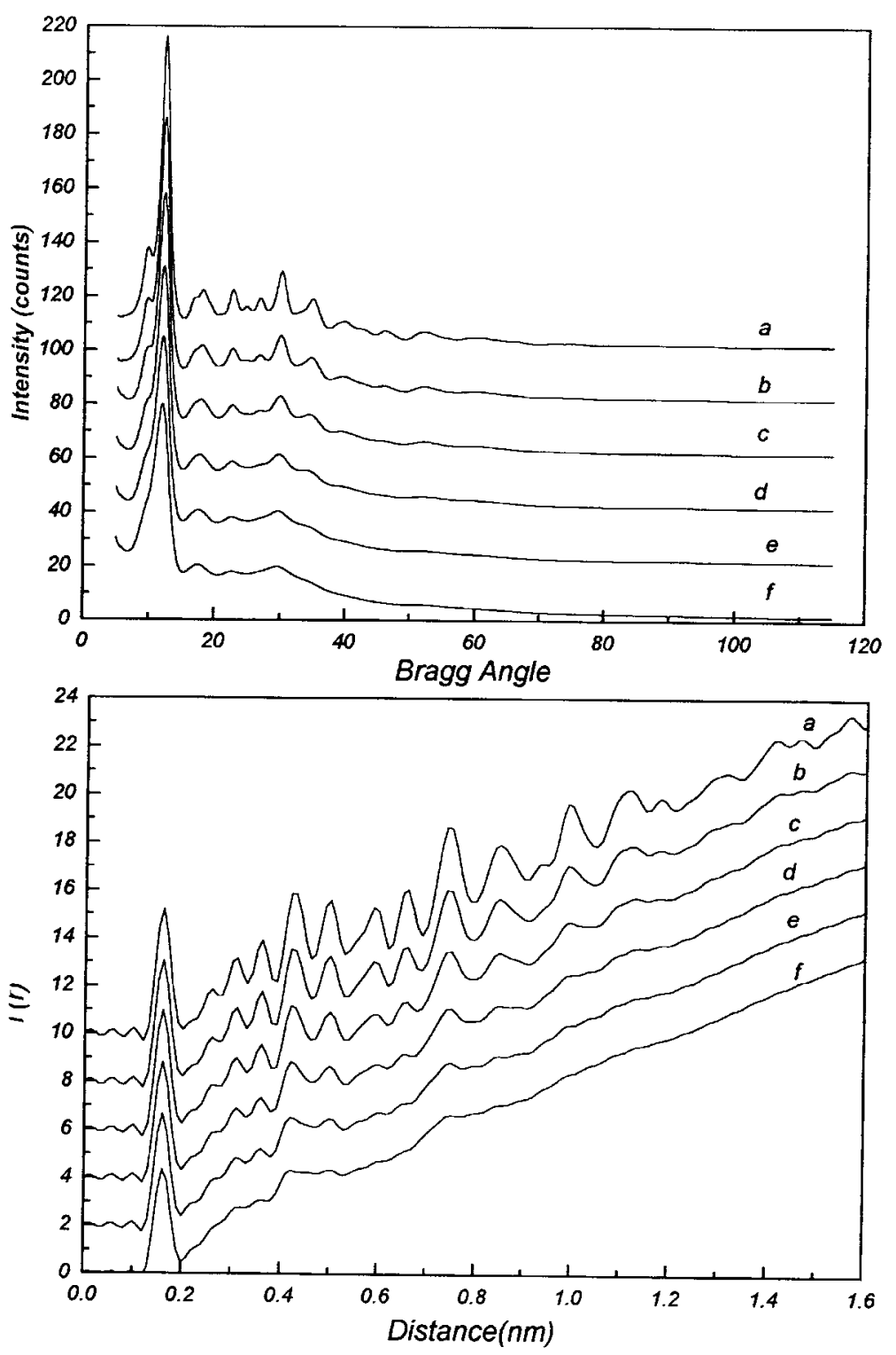

Fig.(2): Simulated XRD patterns and distance correlation functions, T(r), at crystallite size equals $1 \mathrm{~nm}$ and microstrain equals a) 0.01 , b) 0.02 , c) 0.03 , d) 0.04, e) 0.05 or f) 0.06 . 

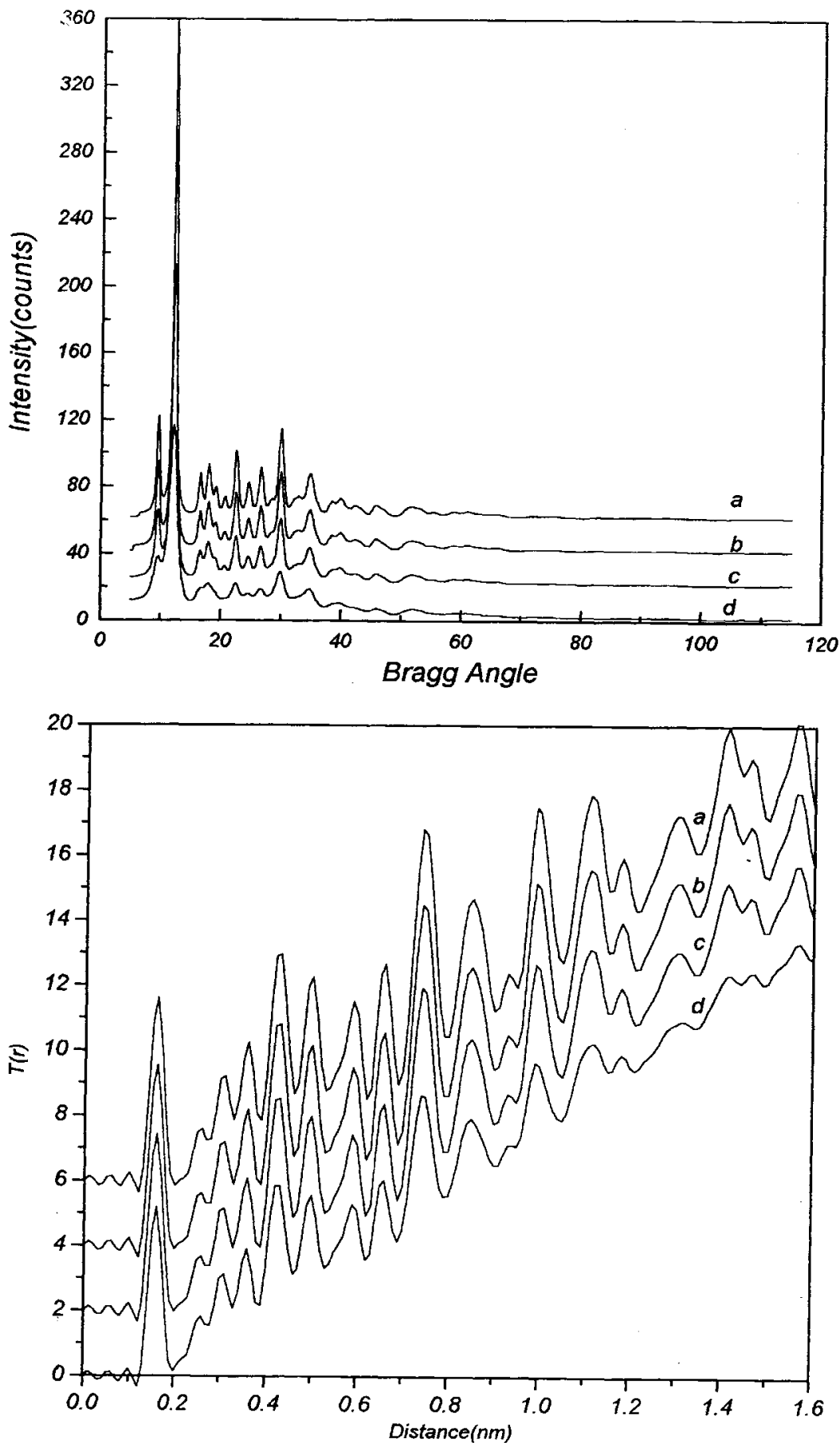

Fig.(3): Simulated XRD patterns and distance correlation functions, T(r), at microstrain equals 0.02 and crystallite size equals a) 4, b) 3 , c) 2 or d) $1 \mathrm{~nm}$. 
part except for peak degradation which is an indication of less order in the network structure[19]. The XRD peak profile are broadened as either the microstrain increases or the crystallite size decreases. For $\mathrm{T}(\mathrm{r})$ profile, in the first case, the broadening is considerable at short- and medium-range up to 1.6 $\mathrm{nm}$. However, in the second case, the broadening of the peaks is predominant mainly at r-range $>0.8 \mathrm{~nm}$ and almost remains with considerable resolution in the shorter range of $r<0.8$. This is because the decrease of crystallite size and increase of microstrain broaden the XRD profile while the distortion of the atomic coordinates are affected mainly by the internal strain and, consequently, the $\mathrm{T}(\mathrm{r})$ profile. The effect of decreasing the crystallite size down to $1 \mathrm{~nm}$ has no pronounced effect as the increase of the internal microstrain up to 0.06. This is in accordance with the finding of Keen and Dove [17] that the similarities between the crystalline and amorphous structure extends over a too short correlation length; of $0.75 \mathrm{~nm}$ in case of HP-tridymite. If one needs to admit crystallite size less than this value, it will be of meaningless, since the lattice parameters $\sim 0515 \pm 0.025 \mathrm{~nm}$. Taken into account the XRD patterns and the $\mathrm{T}(\mathrm{r})$ curves, it was found that crystallite size $\mathrm{M}$ of about $1 \mathrm{~nm}$ and strain $\left(\varepsilon^{2}\right)^{1 / 2}$ $=0.045 \pm 0.015$ are the optimum values.

The profile fitting of $\mathrm{T}(\mathrm{r})$ for this condition is shown in Fig.(4) and the corresponding data are given in Table (1). There are three peaks in the shortrange region at $\mathrm{r}=0.163 \mathrm{~nm}$, for the Si-O bond, $\mathrm{r}=0.261 \mathrm{~nm}$ for the $\mathrm{O}-\mathrm{O}$ bond and $r=0.311 \mathrm{~nm}$ for the $\mathrm{Si}-\mathrm{Si}$ bond. Thus, there are no significant difference between the $\mathrm{T}(\mathrm{r})$ curves of the artificial amorphous-like and the that of the amorphous silica[17]. Homma et al.[20] compared the RDF(r) of amorphous alloys with those of the intermetallics with the same composition. They found that the short rang structure of most of the studied amorphous alloys resembling their crystalline counterparts. So, the short-range structure, i.e., atomic correlation of the first and second coordination shells in the r-range up to about $0.3 \mathrm{~nm}$, matches, at least, qualitatively that of the crystalline counterpart. Thus, the comparative study of the amorphous material with their crystalline counterparts is helpful to understand their short-range structure[20]. 


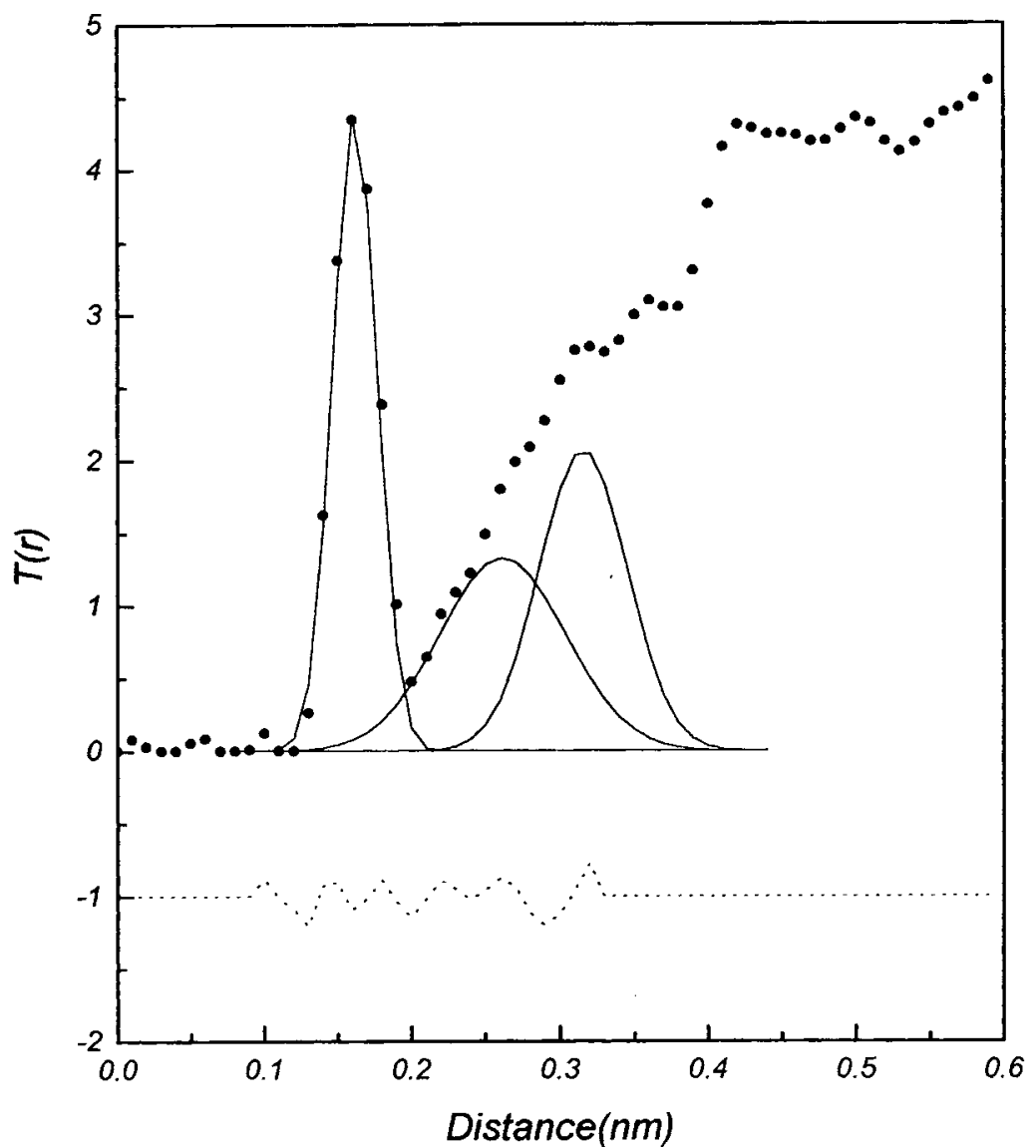

Fig.(4): T(r) curve and fit (solid line) for the first three peaks corresponding to $\mathrm{Si}-\mathrm{O}, \mathrm{O}-\mathrm{O}$ and $\mathrm{Si}-\mathrm{Si}$, respectively. The residuals are displaced by -1 and depicted in the bottom of the figure (dashed line).

Table (1)

Bond lengths, angle and atomic densities $\left(\rho_{0}\right)$ for crystalline $\alpha$-quartz and silica glass (All data were calculated from the $T(r)$ curves).

\begin{tabular}{|l|c|c|c|}
\hline \multirow{2}{*}{} & \multirow{2}{*}{$\begin{array}{c}\alpha-\text { Quartz } \\
\text { Ref. [17] }\end{array}$} & \multicolumn{2}{|c|}{ Silica Glass } \\
\cline { 3 - 4 } & 66.1 & Ref. [17] & This Work \\
\hline$\rho_{o}($ atoms/nm) & 05.7 & 65.4 \\
\hline $\mathrm{Si}-\mathrm{O}(\mathrm{nm})$ & 0.160 & 0.162 & 0.163 \\
\hline $\mathrm{O}-\mathrm{O}(\mathrm{nm})$ & 0.262 & 0.263 & 0.261 \\
\hline $\mathrm{Si}-\mathrm{Si}(\mathrm{nm})$ & 0.311 & 0.310 & 0.311 \\
\hline $\mathrm{O}-\mathrm{Si}-\mathrm{O}\left({ }^{\circ}\right)$ & 109.5 & 108.6 & 106.4 \\
\hline $\mathrm{Si}-\mathrm{O}-\mathrm{Si}\left(^{\circ}\right)$ & 151.0 & 147.0 & 145.1 \\
\hline
\end{tabular}




\section{Conclusions}

One can concluded that: (i) Decreasing crystallite size increases the broadening of the XRD profile, however, it affects $\mathrm{T}(\mathrm{r})$ only in $\mathrm{r}$-range $>0.8$ $\mathrm{nm}$, and (ii) Increasing strain affects the resolution of both XRD profile and $\mathrm{T}(\mathrm{r})$ in the short- and medium-range up to $1.6 \mathrm{~nm}$. On general, decreasing crystallite size down to about $1 \mathrm{~nm}$ and increasing microstrain up to range of $0.045 \pm 0.015$ result in a diffraction pattern similar to that of the amorphous one as well as the a distance (real space) correlation function with the characteristics corresponding to that of the amorphous structure of silica glass. Accordingly, this comparative study is a good verification of the crystallite theory and emphasizes the importance of the microcrystalline approach, that amorphous solid is composed of strained very small crystals of a polymorph. Also, it is worth to mention that, using the full pattern fitting of Rietveld method is a possible approach to study amorphous materials of non-crystalline structure.

\section{References}

1. A.C. Wright, "Experimental Techniques of Glass Science", Edt. G. J. Simmons and O.H. El-Bayoumi, Ceramic Transactions, American Ceramic Society, Chapter 8, (1993).

2. A. Le Bail, C. Jacobni and R. Pape; J. Phys. Coll., C8(1985)163.

3. A. Le Bail,; J. Non-Cryst. Solids, 183(1995)39.

4. A. C. Wright; J. Non-Cryst. Solids, 123(1990)129.

5. L. Lutterotti and P. Scardi; J. Appl. Cryst. 23 (1990) 246.

6. B. E. Warren and B. L. Averbach; J. Appl. Phys., 21 (1950) 595.

7. R. A. Young: "The Rietveld Method", IUCr Monographs on Crystallography, 5 (1993).

8. R. A. Young and D. B. Wiles; J. Appl. Cryst., 15 (1982) 430.

9. A. C. Larson and R. B. Von Dreele: "Generalized Structure Analysis System (GASAS)", LAUR 86-748, Los Alamos National Laboratory (1988).

10. G. Caglioti, A. Paoletti and F. P. Ricci; Nucl. Instrum. Methods, 3 (1958) 223.

11. H. A. Rietveld; J. Appl. Cryst., 2 (1969) 65.

12. P. Scardi, L. Lutterotti and P. Maistrelli; J. Appl. Cryst., 25 (1992) 459.

13. P. Scardi, L. Lutterotti, R. DiMaggoi and P. Maistrelli; Mat. Sc. Fourm, 79-82 (1991) 233.

14. R. K. Nandi, H. K. Kuo, W. Schlosberg, G. Wissler, J. B. Cohen and B. Jr. Crist; J. Appl. Cryst., 17 (1984) 22.

15. Y. Waseda; "The Structure of Non-Crystalline Materials", New York, McGraw-Hill, (1983). 
16. P. H. Gaskell and D. J. Wallis; Phys. Rev. Lett., 76 (1996) 66.

17. D. A. Keen and M. T. Dove; J. Phys. Condens. Matter, 11 (1999) 9263.

18. R. W. Wyckoff, "Crystall Structure”, John Willey\&Sons, Inc., New York, (1963).

19. B. Himmel, J. H. Berber, W. Heyor and W. Blau; J. Mater. Sci., 22 (1987).

20. Y. Homma, K. Sumiyama, K. Suzuki, T. Hihara, H. Amaro and Matsuura; Mater. Trans., JIM, 36 (1995) 816. 